\title{
On the identification of low allele frequency mosaic mutations in the brains of Alzheimer disease patients
}

\author{
Carlo Sala Frigerio ${ }^{\mathrm{a}, \mathrm{b}}$, Pierre Lau ${ }^{\mathrm{a}, \mathrm{b}}$, Claire Troakes ${ }^{\mathrm{c}}$, Vincent Deramecourt ${ }^{\mathrm{d}}$, Patrick Gele ${ }^{\mathrm{d}}$, \\ Peter Van Loo ${ }^{\mathrm{b}, \mathrm{e}, \mathrm{f}}$, Thierry Voet ${ }^{\mathrm{f}, \mathrm{g}, *}$, Bart De Strooper ${ }^{\mathrm{a}, \mathrm{b}, \mathrm{h}, * *}$ \\ ${ }^{a}$ VIB Center for the Biology of Disease, KU Leuven, Leuven, Belgium \\ ${ }^{b}$ Center for Human Genetics, KU Leuven, Leuven, Belgium \\ ${ }^{c}$ Department of Basic and Clinical Neuroscience, Institute of Psychiatry, Psychology and Neuroscience, King's College London, London, UK \\ ${ }^{d}$ Université Lille Nord de France, UDSL, Lille, France \\ ${ }^{e}$ Cancer Research UK London Research Institute, London, UK \\ ${ }^{f}$ Wellcome Trust Sanger Institute, Hinxton, UK \\ ${ }^{g}$ Department of Human Genetics, Laboratory of Reproductive Genomics, KU Leuven, Leuven, Belgium \\ ${ }^{h}$ Department of Molecular Neuroscience, University College London (UCL) Institute of Neurology, London, UK
}

\begin{abstract}
Background: The cause of sporadic Alzheimer's disease (AD) remains unclear. Given the growing evidence that protein aggregates can spread in a "prion-like" fashion, we reasoned that a small population of brain cells producing such "prion-like" particles due to a postzygotic acquired mutation would be sufficient to trigger the disease. Deep DNA sequencing technology should in principle allow the detection of such mosaics.

Methods: To detect the somatic mutations of genes causing AD present in a small number of cells, we developed a targeted deep sequencing approach to scrutinize the genomic loci of APP, PSEN1, and $P S E N 2$ genes in DNA extracted from the entorhinal cortex, one of the brain regions showing the earliest signs of $\mathrm{AD}$ pathology. We also included the analysis of the MAPT gene because mutations may promote tangle formation. We validated candidate mutations with an independent targeted ultradeep amplicon sequencing technique.

Results: We demonstrate that our approach can detect single-nucleotide mosaic variants with a $1 \%$ allele frequency and copy number mosaic variants present in as few as 10\% of cells. We screened 72 $\mathrm{AD}$ and 58 control brain samples and identified three mosaic variants with low allelic frequency ( 1\%): two novel MAPT variants in sporadic AD patients and a known PSEN2 variant in a Braak II control subject. Moreover, we detected both novel and known pathogenic nonmosaic heterozygous variants in PSEN1 and PSEN2 in this cohort of sporadic AD patients.

Conclusion: Our results show that mosaic mutations with low allelic frequencies in AD-relevant genes can be detected in brain-derived DNA, but larger samples need to be investigated before a more definitive conclusion with regard to the pathogenicity of such mosaics can be made.

(C) 2015 The Alzheimer's Association. Published by Elsevier Inc. All rights reserved.
\end{abstract}

*Corresponding author. Tel.: +32-16-33-08-41.

***Corresponding author. Tel.: +32-16-37-31-01; Fax: +32-16-330-827.

E-mail address: Thierry.Voet@med.kuleuven.be (T.V.), bart.destrooper@ cme.vib-kuleuven.be (B.D.S.)

\section{Introduction}

The concepts of somatic disease-causing mutations and of mosaic genomic heterogeneity are well known in the etiology of cancer [1-3]. Recently, several studies have highlighted the role of such acquired mutations as 
pathogenic drivers for neurodevelopmental diseases [4-7]. The possibility that mosaic mutations contribute to neurodegenerative diseases should also be considered [811]. Indeed, neurons accumulate a wide spectrum of somatic mutations, in the forms of single nucleotide variants (SNVs), insertion/deletions (indels), retrotranspositions, copy number variants (CNVs), and wholechromosomal aneuploidies [4,5,12-14]. Although the mutation rate of human cells varies for different kind of mutations and for different tissues, a rate of $1 \times 10^{-10} \mathrm{de}$ novo point mutations per base per cell cycle is a reasonable estimate $[15,16]$, implying approximately one new mutation per cell division. The brain contains $\sim 10^{11}$ neurons and about a similar number of nonneuronal cells [17], thus it is easily conceivable that pathogenic mutations may arise de novo in a mosaic fashion during its ontogenesis. Depending on the time point of the mutation appearance in the cell lineage tree descending from the zygote, the sequencing of DNA isolated from blood may only exceptionally detect such mutation [18] (Fig. 1). This explains why this potentially important phenomenon has not been systematically investigated for Alzheimer's disease (AD).

Most AD patients are sporadic (SAD), i.e., characterized by a late onset and unclear familial inheritance. The biochemical and clinical features of SAD resemble those of familial AD (FAD), which is characterized by a clear autosomal dominant inheritance of causative mutations in mainly three genes (APP, PSEN1, and PSEN2) $[19,20]$. Growing evidence that protein aggregates of $A \beta$ or Tau (encoded by MAPT gene) can spread in the brain and act as local initiators of further aggregation of normal proteins in a "prion-like" fashion [21-25], provides a mechanistic framework to understand how somatic mutations in the brain could spark neurodegenerative disease. De novo mosaic mutations of AD-relevant genes would create a nidus of mutant cells mixed between normal cells that would continuously produce and release proaggregating proteins. Such aggregates could act as seeds for further protein aggregation at sites distal from their origin (Fig. 1).

Detection of low-grade mosaic mutations has been hindered by the low sensitivity of classical Sanger sequencing, which allows the detection of mosaic mutations only with an allelic frequency of at least $20 \%$ [26]. Recent attempts to identify mosaic pathogenic mutations in Parkinson's disease used high-resolution melting analysis, which allows the detection of mutations with $5 \%$ to $10 \%$ allelic frequency [11]. Here, we deep sequenced DNA libraries enriched for AD-relevant genes to achieve high sequencing depth, followed by an amplicon ultradeep sequencing validation: this approach enabled the detection of mosaic SNVs having an allelic fraction as low as $1 \%$.

\section{Materials and methods}

\subsection{Samples}

Small blocks $(\sim 100 \mathrm{mg})$ of entorhinal cortex were obtained from Lille NeuroBank (BB-0033-00030) and
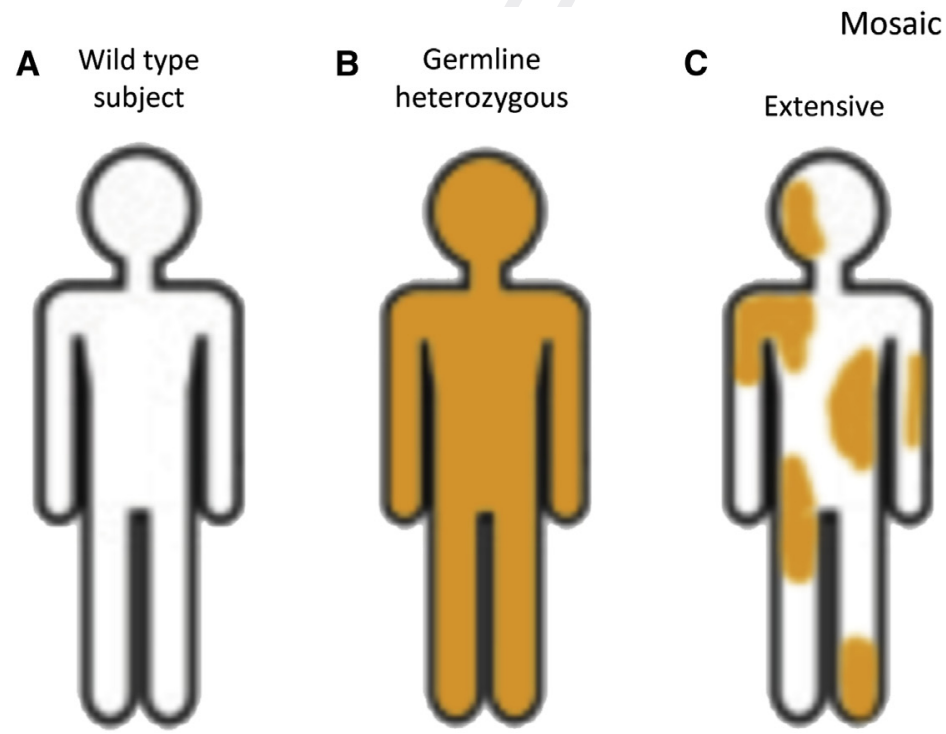

\section{Mosaic heterozygous}

D

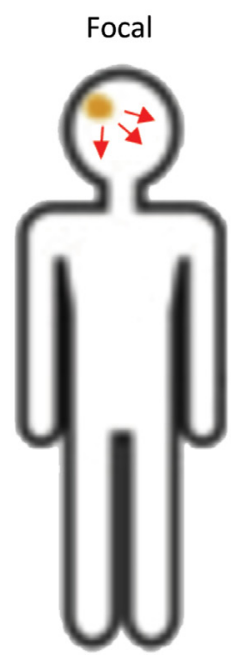

Fig. 1. Somatic mutations and hypothesis of pathology spread in sporadic Alzheimer's disease (AD). An inherited mutation will be carried by all cells of a human body (B), this is the typical case of a familial AD patient. In case of mutations arising in a postzygotic stage, an individual will be a genetic mosaic Q19 for such mutation, with cells either carrying the mutation (orange) or not (white). Depending on the developmental time point of the appearance of the mutation, genetic mosaics can be either extensive (C), with mutant cells appearing in several organs/tissues, or focal (D), when mutant cells are localized in a single organ/ tissue. Our working hypothesis is that some sporadic AD patients are focal mosaics for mutations in AD-relevant genes appearing in brain cells. Amyloid beta $(A \beta)$ and/or tau aggregates produced locally as consequence of the mosaic mutation can then spread (red arrows in (D) and seed further aggregation in other brain areas in a "prion-like" fashion, thus leading to full blown AD. 
from the London Neurodegenerative Diseases Brain Bank. This study was approved by the KU Leuven ethical commission. Plaque burden and Tau tangles were scored according 5 to CERAD parameters and to Braak staging, respectively. Brain samples showing a Braak stage of up to III were included in the "non-AD" group. The "non-AD" group consisted mainly of subjects showing mild ageing processes, consistent with the respective age of death.

\subsection{DNA isolation}

Genomic DNA (gDNA) was isolated from 50 to $100 \mathrm{mg}$ of tissue. Briefly, frozen tissue was mechanically crushed and digested overnight with protease K. Digested samples were treated with RNase A (Qiagen, Venlo, The Netherlands). Subsequently, DNA was isolated with phenol:chloroform:isoamyl alcohol, washed with chloroform:isoamyl alcohol and precipitated with cold $100 \%$. The DNA pellet was washed with $70 \%$ ethanol, air-dried, and reQ6 suspended in TE buffer. Double-stranded DNA content was Q7 assayed using Qubit dsDNA BR assay with a Qubit 2.0 Fluorometer (both from Life Technologies, Gent, Belgium). DNA isolation and quantification were carried out in a laboratory separated from the sites where sequencing libraries were prepared, to minimize the contamination of tissue samples and DNA stocks.

\subsection{Custom library enrichment for region of interest}

A SeqCap EZ Choice Library (NimbleGen, Roche NimbleGen, Madison, WI) was designed to target the genomic regions of APP (chr21:27242859-27553138), PSEN1 (chr14:73593141-73700399), PSEN2 (chr1:227048271227093804), and MAPT (chr17:43961646-44115799), including $10 \mathrm{~kb}$ both upstream and downstream of each locus. For MAPT, we also included regions specific for the alternate assembly of chr17_ctg5_hap1 (chr17_ctg5_ hap1:762280-895830). All genomic coordinates refer to the human genome build hg 19 .

\subsection{Library preparation, enrichment, and deep sequencing}

For library preparation, $3 \mu \mathrm{g}$ of gDNA (corresponding to $\sim 461,538$ copies of diploid genomes, based on an average human diploid cell DNA content of $6.5 \mathrm{pg}$ ) was sheared by sonication and indexed libraries were prepared using the TruSeq DNA kit (Illumina, San Diego, CA). Pools of 10 libraries were enriched with the SeqCap probeset described previously, following manufacturer's recommendations. Each enriched-pool was paired end $(2 \times 100 \mathrm{bp})$ sequenced using a lane of HiSeq2000 (Illumina). To ensure the correct assignment of sequences to each of the pooled samples, indexes had at least three different nucleotides between each other, and sequence demultiplexing was allowed a maximum of one mismatch in the index. Library preparation, enrichment, and sequencing were carried out in the
UZ Genomics Core facility, following strict rules for preand post-PCR rooms.

\subsection{Data analysis}

Raw sequencing data (FASTQ files) were aligned to the hg19 reference genome using BWA (version 0.6.2) [27]. Regions of interest (ROIs) were extracted from the SAM files Q8 using samtools (version 0.1.18) [28]; sequences were then realigned around indels and base qualities were recalibrated using GATK (version 2.0-39-gd91f72) [29]. Variant calls were made using Varscan 2.3.2 [30] on single-sample samtools mpileup files and annotated using Annovar (version 2012May25) [31]. Variants are reported according to the following transcripts: NM_000484 (APP), NM_001123066, and NM_005910 (MAPT), NM_000021 (PSEN1), NM_000447 (PSEN2). CNVs were analyzed using Varscan and the DNAcopy (version 1.36.0) R package. Data were analyzed using the free statistical software $\mathrm{R}$ (http://www.r-project.org/).

\subsection{Amplicon deep sequencing}

Primers were designed using Primer3 (http://bioinfo.ut. ee/primer3-0.4.0/), excluding primers overlapping the positions of known SNPs (as obtained by UCSC genome Q9 browser, track common SNPs [138]). Primers were synthesized by IDT (Leuven, Belgium). Amplicons were prepared using HotStar HiFidelity Polymerase kit (Qiagen) following the manufacturer's recommendations. PCR reactions were carried out for 25 cycles, using $25 \mathrm{ng}$ of template DNA (corresponding to $\sim 3846$ copies of diploid genomes). Amplicons were analyzed on $2 \%$ agarose gels stained with GelGreen (Biotium, Hayward, CA) and specific bands were cut and purified using QIAquick gel extraction kit (Qiagen). Purified amplicons were quantified with Qubit dsDNA HS assay (Invitrogen) and pooled. Individual pools were used to prepare indexed sequencing libraries and sequenced on a MiSeq (Illumina) using paired-end 300 bp reads. FASTQ files were aligned to the hg 19 reference genome using BWA-MEM algorithm of BWA, mutation calling was ${ }_{110}$ performed using samtools mpileup and Varscan. To minimize risks of contamination, amplicons were prepared and sequenced in different laboratories from those where DNA had been isolated and where HiSeq libraries were prepared.

\subsection{Sanger sequencing}

Primer design and amplicon preparation were performed as described previously. The VIB Genetic Service Facility (http://www.vibgeneticservicefacility.be/) performed Sanger sequencing of the purified amplicons.

\subsection{Quantitative PCR}

The copy number of $A P P$ locus was assessed by quantitative PCR using predesigned TaqMan Copy number assays 
(Hs01180853_cn, Hs00525904_cn, Hs05547973_cn) from Applied Biosystems (Foster City, CA). qPCR reactions were assembled in 96-well plates according to manufacturer's instructions using TaqMan Genotyping Master Mix (Applied Biosystems) and 20 ng of template DNA/ reaction. Assays were run in technical quadruplicates on a Lightcycler LC480 (Roche Diagnostics, Basel, Switzerland).

\section{Results}

\subsection{Targeted deep-sequencing allows the detection of mosaic mutations with $1 \%$ allele frequency}

The detection of low-grade mosaic variants is a major methodological challenge, critically relying on high sequencing depth to correctly call a variant over a large number of wild-type sequences and to discriminate the variant from noise due to sequencing errors and read misalignments [32]. To maximize the coverage across our ROIs we enriched our libraries using a custom-designed probe set for the genomic loci of PSEN1, PSEN2, APP, and $M A P T$ genes, including $10 \mathrm{~kb}$ up- and downstream for each locus, to gather enough information for both SNV and CNV calling. Available solutions to read misalignments are local realignment (with tools such as GATK IndelRealigner [29]) and base quality recalibration (with algorithms such as BAQ recalculation implemented by samtools mpileup [33]). Sequencing errors can be estimated using several computational methods, here we use Varscan 2.0 [30] as it was found to excel in low-grade mosaic mutations calling [32].

To establish our workflow and benchmark our method, we analyzed a series of "synthetic mosaics" prepared by mixing gDNA of fibroblasts carrying a heterozygous APP E682K mutation ( $\mathrm{C}>\mathrm{T}$ chr21:27269905) with gDNA carrying a homozygous wild-type allele (Supplementary Fig. 1A). As expected, on average more than half of the total reads were aligned to the ROI $(52.3 \% \pm 5.9 \%$, avg \pm standard deviation or $\mathrm{SD})$, allowing for high sequencing depth (average coverage $2735 \mathrm{X} \pm 429 \mathrm{X} \mathrm{SD}$; average $82.6 \% \pm 3.9 \%$ SD of ROI covered at $\geq 1000 \times$ ). To maximize the sensitivity and specificity of mosaic SNV calling, we tested several pipelines combining the modules of GATK and mpileup BAQ with Varscan (Fig. 2). The position of the pilot APP mutation was highly covered in all "synthetic mosaic" samples ( $3572 \pm 759$ reads, avg \pm SD) and could be readily identified by several calling pipelines down to the $1 \%$ "synthetic mosaic" (actual observed mutant allele frequency of $0.94 \%-0.96 \%$ ), whereas the $0.5 \%$ "synthetic mosaic" could not be distinguished from the $0 \%$ sample (Fig. 2A). A high base-quality cut-off [30] in Varscan in combination with BAQ recalculation failed to call the heterozygous mutation. To evaluate the sensitivity of our method, we considered all the heterozygous SNVs in the original APP mutant DNA sample which were absent in the admixed one (therefore following the same behavior as the pilot mutation in the mixed samples, Supplementary Fig. 1A) and which were sequenced at $\geq 1000 \times$. Sensitivity and accuracy, as measures of the fraction of mosaic variants called and the correctness of the observed mutant allele frequency, respectively, were strikingly different across the pipelines, the best performing being GATK-BAQ-V15 and noGATK-BAQ-V15 (Fig. 2B). Both pipelines were able to detect all the synthetic mosaic variants at $1 \%(\mathrm{n}=38$ and 36 , respectively $)$ at high accuracy (observed mutant allele frequency $1.6 \% \pm 0.7 \%$ SD) (Fig. 2B). The accuracy for the "synthetic mosaic" variants at $0.5 \%$ was not satisfactory, as they did not recover all the variants (for a variant to be called in a "synthetic mosaic" sample, its observed allele frequency had to be higher than the average observed allele frequency in the $0 \%$ sample, for the same pipeline) (Fig. 2B, right panel).

To evaluate specificity, we reasoned that genomic positions sequenced at very high depth $(\geq 1000 \times)$ and displaying no mutant bases in both original DNAs should show no mutant bases in the mixed samples as well. Mutations called at these positions in the mixtures would then constitute false positive (FP) calls. With all the tested pipelines we identified a very high number of FP calls having very low allelic frequencies, as expected (Supplementary Table 1, Supplementary Fig. 2 and Fig. 2C). In general, the number of FP rose steeply below the $1 \%$ mark, explaining why the $0.5 \%$ "synthetic mosaic" could not be distinguished from background errors (Fig. 2A and B). BAQ recalculation greatly reduced the number of FP, in particular those few with allelic frequency greater than $1 \%$ (Fig. 2C, compare left and middle panels).

Taken together, these results show that the GATK-BAQV15 analysis pipeline is able to detect the "synthetic mosaic" variants with an alternate allele frequency as low as $1 \%$, with a manageable trade-off of FP calls.

\subsection{Identification and validation of low-grade mosaic variants in brain}

We next analyzed gDNA isolated from entorhinal cortex samples of a cohort of 72 SAD and 58 nonAD control subjects (demographics are provided in Supplementary Fig. 3 and Supplementary Table 2). As expected, capture efficiency varied between different experiments $(41 \% \pm 20 \%$ SD of the total sequences aligned to ROI) but the average sequencing depth across our ROI was in all instances high enough for the detection of low-grade mosaic mutations $(2153 \mathrm{X} \pm 985 \mathrm{X}$, avg $\pm \mathrm{SD} ; 85.3 \% \pm 8.6 \%$ avg \pm SD of ROI covered at $\geq 1000 \times$ ). We determined all variants using the established variant calling pipeline, and selected for further analysis the nonsynonymous variants with an observed allelic frequency $\geq 0.9 \%$ 

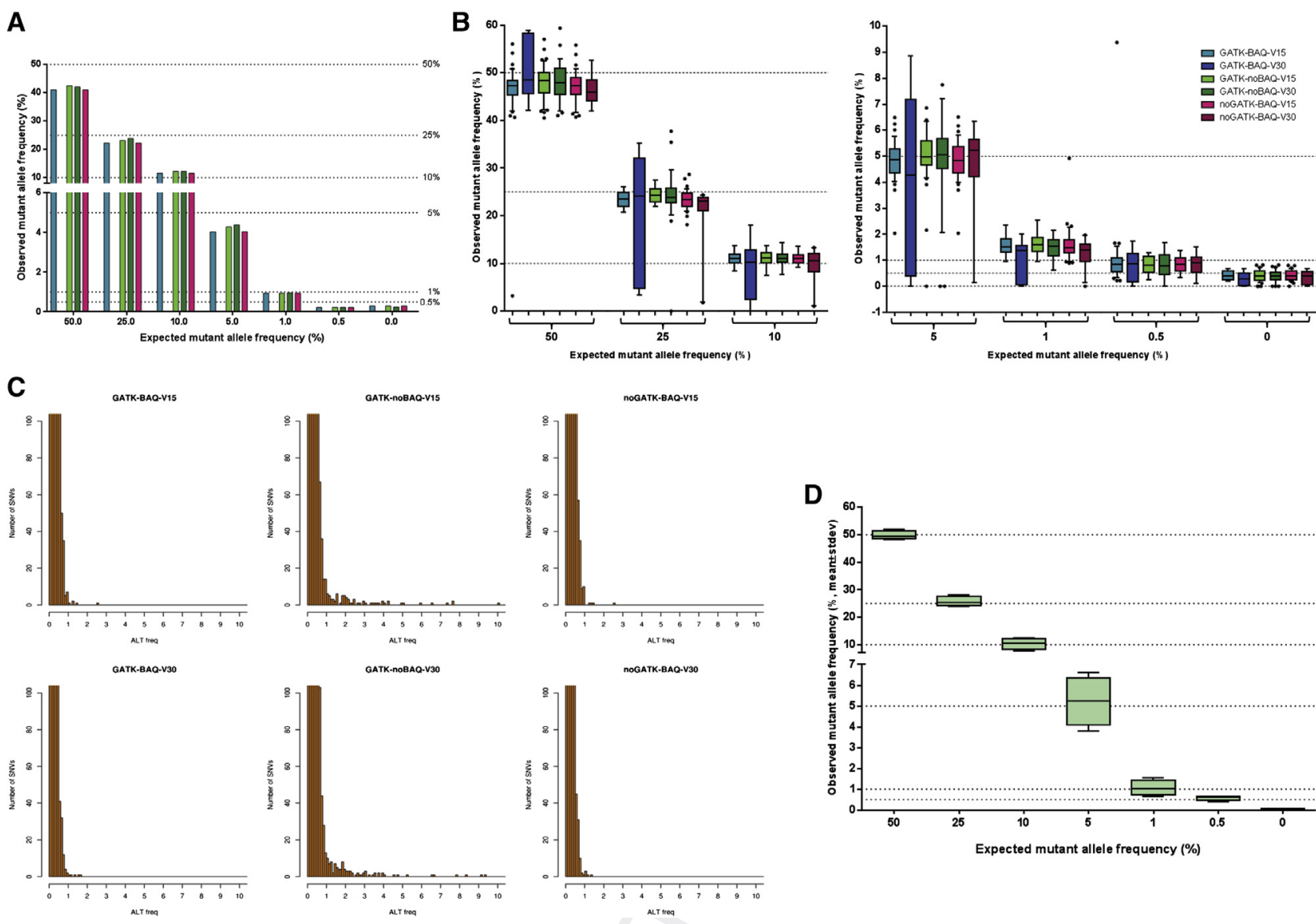

Fig. 2. Deep sequencing of capture-enriched libraries allows the detection of $1 \%$ mosaic mutations and MiSeq targeted amplicon sequencing allows the detection of mosaic variants with $0.5 \%$ mutant allele frequency. (A) We tested several combinations of software to call mutations in the sequenced libraries, i.e., the GATK indel-realigner/base-recalibration (GATK), the mpileup with (BAQ) and without (noBAQ) BAQ recalculation and the Varscan quality filter (V15 and V30 for base quality thresholds of 15 and 30, respectively). We used a defined series of serial dilutions of DNA bearing an $A P P$ single nucleotide variant (SNV) (C > T at position chr21:27269905) and wild-type DNA. For each combination of software tools (pipeline), the observed mutant allele frequency of the pilot APP SNV is plotted against the expected mutant allele frequency. (B) To further assess sensitivity and accuracy of the pipelines, we analyzed the SNVs called heterozygous in the APP mutant DNA and wild type in the admixed DNA. For each dilution sample (indicated by the expected mutant allele frequency on the $\mathrm{x}$ axis), we plot the observed mutant allele frequency of the SNVs analyzed by each pipeline ( $\mathrm{n}=38$ for GATK-BAQ-V15, $\mathrm{n}=8$ for GATK-BAQ$\mathrm{V} 30, \mathrm{n}=41$ for GATK-noBAQ-V15, $\mathrm{n}=29$ for GATK-noBAQ-V30, $\mathrm{n}=36$ for noGATK-BAQ-V15, $\mathrm{n}=12$ for noGATK-BAQ-V30). Boxes extend from the 25 th to the 75 th percentile with whiskers extending to 10 th and 90 th percentile, data points outside the whiskers are represented with dots. The horizontal line in each box represents the median. The plot has been divided in two panels to allow better readability. (C) To evaluate the specificity of each calling pipeline, we plotted the sum of the false positive (FP) SNV calls detected in the five dilution samples (number of SNVs, y axis) for each bin (0.1\%) of mutant allele frequency (ALT freq, x axis); axes are zoomed to 10\% (x axis) and 100 (y axis). The full graphs are reported in Supplementary Fig. 2. (D) To validate candidate mosaic mutations identified by HiSeq sequencing of capture-enriched libraries, we used amplicon sequencing on a MiSeq. This latter method was benchmarked by mixing the genomic DNA of four individuals with mutations at positions chr1:227083249 (PSEN2 gene), chr14:73653568 (PSEN1 gene), chr17:44067341 (MAPT gene), and chr21:27269905 (APP gene), respectively, wild-type DNA to prepare templates with different amounts of mutant alleles (25\%, 10\%, $5 \%, 1 \%$, and $0.5 \%$ ). Parental DNAs (50\% and $0 \%$ mutant alleles, respectively) and mixed samples were PCR amplified and sequenced on a MiSeq. For each dilution ( $\mathrm{n}=4$ for dilution step), the observed mutant allele frequency is plotted as a box plot (same as in (B)). In each case the observed frequency closely matches the expected frequency (dotted lines). Mutations present at $0.5 \%$ mutant allelic frequency could be called against the background.

and with a coverage $\geq 900$. Based on the analysis of the "synthetic mosaic" samples, an allelic frequency cutoff of $0.9 \%$ provides very high sensitivity $(>97 \%)$ with an acceptable trade-off of FP calls $(4.2 \pm 3.6$, average \pm SD, FP calls per sample of 130,744 potential positions; Supplementary Fig. 4). We also note that using a minimum coverage of $900 \times$ instead of $1000 \times$ leads to one extra FP calls at a $0.9 \%$ allelic frequency cut-off $(4.2 \pm 3.6 \mathrm{FPs} / \mathrm{sample}$ versus
$3.2 \pm 2.8$ FPs/sample, respectively). Excluding a series of known nonpathogenic MAPT polymorphisms (P202L，Q230R，D285N，V289A，R370W，Y441H, S447P), we retained a total of 128 variants. Of these, 107 had a mutant allele frequency compatible with a mosaic nature $(0.9 \% \leq$ frequency $<40 \%)$, whereas the remainder had allele frequencies between $40 \%$ and $60 \%$ and were therefore inferred to be heterozygous (Supplementary Table 3 and Fig. 3). 
A

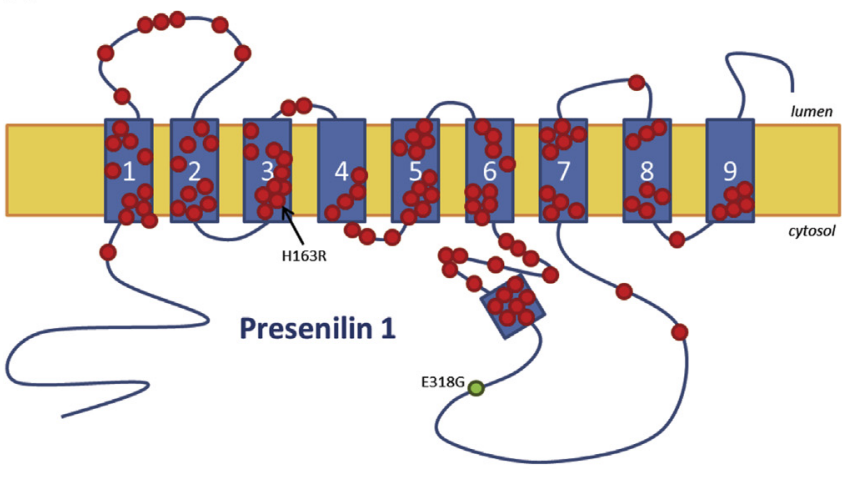

C

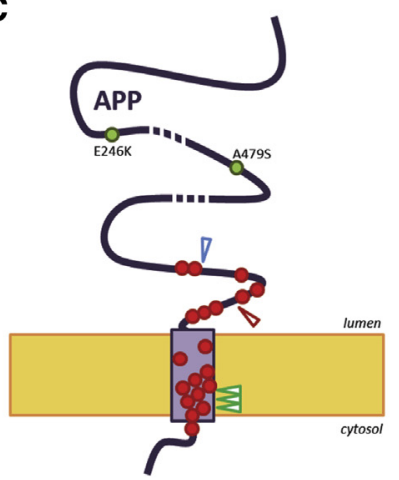

B

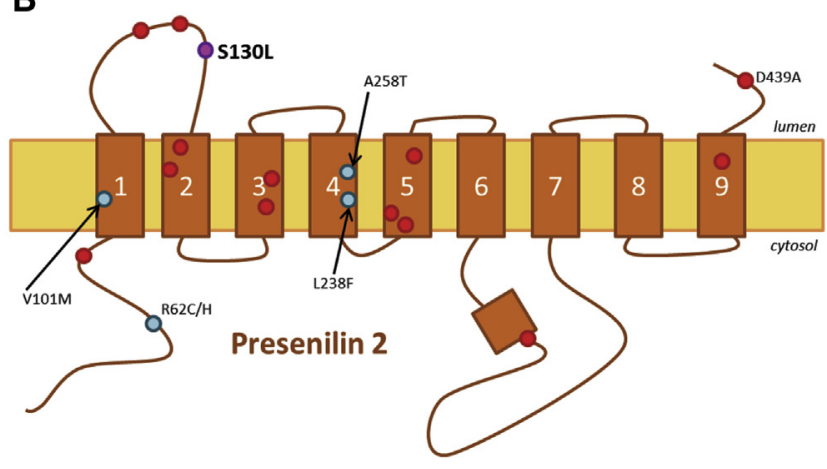

D

Fig. 3. Mosaic and heterozygous nonmosaic variants found. We illustrate the positions of mosaic mutations (purple dots) and of novel mutations (blue dots) found in the present study in relation to previously described pathogenic mutations (red dots) and known nonpathogenic variants (green dots) in PSEN1 (A), PSEN2 (B), APP (C), and Tau (D). Mutations found in this study are named, mosaic ones are in bold. Numbered blue boxes in (A) and (B) represent transmembrane domains of PSEN2, green boxes in (D) represent the microtubule binding domains of Tau. The blue, red, and green triangles in (C) indicate $\beta-, \alpha-$ and $\gamma$-secretase cleavage sites, respectively.

By extrapolating the number of FP calls per sample counted in our pilot experiment to the exonic regions captured in these experiments $(18,836$ nucleotides), we expected 0.1 to $1.1 \mathrm{FP}$ calls per sample. Thus we anticipated between $\sim 12$ and $\sim 145 \mathrm{FP}$ calls in the analysis of the full cohort. Therefore, to validate candidate mosaic variants, we applied an independent amplicon ultradeep resequencing approach. Amplicons were sequenced on an Illumina MiSeq to very high depth $(12,455 \mathrm{X}$ to $324,885 \mathrm{X}$ depend- ing on the amplicon, on average $111,485 \mathrm{X} \pm 81,033 \mathrm{X}$ SD) and analyzed with the GATK-BAQ-V15 pipeline (Supplementary Table 3). To assess the sensitivity and accuracy of this approach, we diluted gDNA of four individuals bearing a different heterozygous mutation confirmed by Sanger sequencing with wild-type gDNA, covering a wide range of mutant allele frequencies (Fig. 2D). In all cases, the observed mutant allele frequencies closely matched the expected ones (Pearson $\mathrm{r}=0.996$,

Table 1

Mosaic mutations found in our cohort

\begin{tabular}{|c|c|c|c|c|c|c|c|c|c|c|c|}
\hline Subject & Group & Gene & Mut & rsID & Notes & Familiarity & Variant & $\begin{array}{l}\text { ALT } \\
\text { frequency } \\
\text { HiSeq }\end{array}$ & $\begin{array}{l}\text { ALT } \\
\text { frequency } \\
\text { MiSeq }\end{array}$ & MAF & Polyphen2 \\
\hline BBN_9943 & $\mathrm{AD}$ & MAPT & Q124K & & NOVEL & No & $C>A$ chr17:44055803 & 1.1 & 1.0 & n.a. & $\begin{array}{l}\text { Probably } \\
\text { damaging }\end{array}$ \\
\hline BBN_9959 & $\mathrm{AD}$ & MAPT & $\begin{array}{l}\text { S735A } \\
\quad(\mathrm{S} 400 \mathrm{~A})\end{array}$ & & NOVEL & $\begin{array}{l}\text { None } \\
\quad \text { recorded }\end{array}$ & $\mathrm{T}>\mathrm{G} \operatorname{chr} 17: 44101409$ & 1.0 & 0.7 & n.a. & $\begin{array}{l}\text { Probably } \\
\text { damaging }\end{array}$ \\
\hline BBN_16242 & $\mathrm{CT}$ & PSEN2 & S130L & rs63750197 & Known & $\begin{array}{l}\text { None } \\
\quad \text { recorded }\end{array}$ & $\mathrm{C}>\mathrm{T}$ chr1:227073271 & 5.7 & 1.6 & $>0.1$ & $\begin{array}{l}\text { Possibly } \\
\text { damaging }\end{array}$ \\
\hline
\end{tabular}

NOTE. For each of the three validated mosaic mutations, we report the information on the mutation bearing subject (diagnostic group, family history of AD) and the information on the mutation (amino acid change, nucleotide change, genomic position, alternate allele frequency observed with HiSeq sequencing of the enriched library and the one observed with MiSeq sequencing of the targeted amplicon, MAF in the population (\%) according to dbSNP and the effect predicted by Polyphen2). Mutation MAPT S735A (Tau-G numbering) is equivalent to MAPT S400A (Tau-F numbering, 441 amino acid isoform). 
Fig. 2D). Importantly, mutations with frequencies as low as $0.5 \%$ could be readily called against background signal (observed mutant allele frequencies of $0.58 \% \pm 0.12$ (avg $\pm \mathrm{SD})$ for variants expected to be $0.5 \%$ versus $0.05 \% \pm 0.03$ (avg $\pm \mathrm{SD}$ ) when $0 \%$ was expected, Fig. 2D), highlighting the superb sensitivity and accuracy of the validation assay.

Only three out of the 107 candidate variants were confirmed in the validation assay (Table 1 and Fig. 3). We considered a variant to be validated if its observed mutant allele frequency was above the sensitivity of detection of MiSeq, estimated to be $0.5 \%$ from Fig. 2D. Subject BBN_9943, who died at 90 years old diagnosed with $\mathrm{AD}$ at Braak stage VI and CERAD plaque score C, showed a novel MAPT Q124K mutation present at $1.0 \%$ allelic frequency (concordant with the observed $1.1 \%$ frequency of the HiSeq sequencing). A control subject, BBN_16242, who died at 90 years old was diagnosed with mild AD-type changes (modified Braak stage II) and mild amyloid angiopathy, and showed a known PSEN2 S130L mutation present at $1.6 \%$ allelic frequency (in contrast to the $5.7 \%$ observed alternate allele frequency reported by HiSeq sequencing). Subject BBN_9959, an AD patient with an apparent age of onset at 85 years and deceased at 91 years, showed a novel MAPT S735A (S400A in the Tau-F isoform) present at $0.7 \%$ allele frequency (compared with a $1.0 \%$ frequency observed by HiSeq sequencing).

The candidate mosaic $\mathrm{C}>\mathrm{T}$ variant at chr1:227073271 (PSEN2 S130L mutation) discovered in 13 different subjects in the initial screen, was found to be a FP call in 12 of them following validation. Contamination from the sample carrying the heterozygous PSEN2 S130L variant is unlikely, as this sample and those carrying the candidate variants were prepared far apart in time and sequenced in different sequencing runs. More conclusively, three nearby heterozygous variants in the heterozygous $\mathrm{S} 130 \mathrm{~L}$ carrier (allele frequencies of $46.14 \%, 45.42 \%$, and $46.71 \%$, respectively) were not found with compatible allele frequencies in the mosaic S130L carrier (below the defined limit of $0.9 \%$ for the detection of mosaic variants in HiSeq sequences for the first two and $99.5 \%$, for the last one). Thus the detection of this particular mosaic variant cannot be explained by contamination with the DNA stock or sequencing library from the heterozygous sample. These results indicate that our targeted deep sequencing method is able to identify candidate mosaic variants but with a high cost of false positives. Amplicon ultradeep resequencing is therefore absolutely necessary for validation.

\subsection{Validation of heterozygous variants confirms their likely germline nature}

From the initial targeted deep sequencing, we also identified 21 heterozygous variants (Table 2). Four vari- ants are known risk factors (PSEN1 E318G, MAPT V224G, MAPT S427F and MAPT A469T) [34,35] and one is nonpathogenic (MAPT IVS10 + 29) [36]. Moreover, two APP mutations (E246K and A479S) are located outside the $A \beta$ region and are unlikely pathogenic. All remaining variants were validated by classical Sanger sequencing and further investigated in other tissues when possible, in an effort to clarify their germline or mosaic nature.

For one subject, carrying PSEN2 A258T, DNA extracted from blood cells was available, which allowed unequivocal confirmation of the germline nature of the variant. In all the other instances, only DNA from other brain areas was available; here Sanger sequencing analysis showed that these variants were present at heterozygous frequency and are therefore likely germline. Four of such variants, three found in AD subjects with no family history (MAPT A90V, PSEN2 V101M, and PSEN2 L238F) and one in a control subject (MAPT P4T), are novel. The remaining five variants (PSEN1 H163R, PSEN2 R62C, PSEN2 R62H, PSEN2 S130L, and PSEN2 D439A) have been previously described in association with FAD; all of them except the latter were found in SAD patients.

\subsection{Targeted deep sequencing allows the detection of mosaic CNVs present in as low as $10 \%$ of cells}

To test the sensitivity and specificity of our method for identifying mosaic CNVs, we analyzed a set of "synthetic CNV mosaic" samples obtained by mixing DNA isolated from amniocytes of a trisomy 21 (T21) fetus with DNA from euploid fibroblasts $(75 \%, 50 \%, 10 \%, 5 \%, 1 \%$, and $0.5 \%$ T21 DNA, respectively, Supplementary Fig. 1B), taking advantage of the fact that the APP locus is on chromosome 21.

Data analysis made use of the ratio of normalized sequencing depth (on a $\log$ scale, $\log R$ ) and of the frequency of the SNP alternate allele (B allele frequency, BAF), which are well-established data sources used for CNV analyses with SNP arrays [37]. In the 100\% T21 DNA, trisomy 21 could be readily detected by analyzing the LogR data (Fig. 4A). In addition, the BAF data for this sample show SNPs with allele frequencies of $\sim 66 \%$ and $\sim 33 \%$, as expected [38] (Fig. 4B). In contrast, both $\operatorname{LogR}$ and BAF demonstrated normal values for PSEN2, PSEN1, and MAPT in the $100 \%$ T21 sample, consistent with the diploid nature of these loci (Supplementary Fig. 5), thus confirming the accuracy of the method. $\log \mathrm{R}$ analysis was furthermore able to identify decreasing grades of mosaicism, down to $10 \%$, across the APP locus (Fig. 4A), confirmed by BAF data analysis (Fig. 4B). As a validation, we tested a qPCR-based CNV detection method with three different commercially available Taqman CNV assays; the sensitivity limit was 25\% T21 DNA (Fig. 4C). Thus, we conclude that the enriched library-deep sequencing method and data 
Table 2

Heterozygous variants found in our cohort

\begin{tabular}{|c|c|c|c|c|c|c|c|c|c|c|c|}
\hline Subject & Group & Gene & Mut & rsID & Notes & Familiarity & Variant & $\begin{array}{l}\text { ALT frequency } \\
\text { HiSeq }\end{array}$ & MAF & Polyphen 2 & Other tissues \\
\hline C08-10048 & $\mathrm{AD}$ & MAPT & A90V & & NOVEL & No & $\mathrm{C}>\mathrm{T}$ chr17:44051799 & 45.7 & n.a. & Benign & n.a. \\
\hline BBN_10196 & $\mathrm{AD}$ & PSEN2 & V101M & & NOVEL & No & $G>A$ chr1:227071565 & 43.4 & n.a. & Probably damaging & cer \\
\hline BBN_9967 & $\mathrm{AD}$ & PSEN2 & L238F & & NOVEL & No & $\mathrm{C}>\mathrm{T}$ chr1:227076675 & 53.5 & n.a. & Probably damaging & cer \\
\hline BBN_3761 & $\mathrm{AD}$ & PSEN2 & $\mathrm{R} 62 \mathrm{C}$ & rs150400387 & Known & Brother had AD & $\mathrm{C}>\mathrm{T}$ chr1:227071448 & 55.3 & $>0.1$ & Possibly damaging & cer \\
\hline C09-20305 & $\mathrm{AD}$ & PSEN1 & H163R & rs63750590 & Known & No & $A>G$ chr14:73653568 & 49.4 & NA & Possibly damaging & hippo, cer \\
\hline BBN_9975 & $\mathrm{AD}$ & PSEN2 & $\mathrm{R} 62 \mathrm{H}$ & rs58973334 & Known & No & $\mathrm{G}>\mathrm{A}$ chr1:227071449 & 45.7 & 1.8 & Benign & cer \\
\hline BBN_9975 & $\mathrm{AD}$ & APP & E246K & rs147485129 & $\begin{array}{l}\text { Outside of } \mathrm{Ab} \text { region } \\
\text { outside of } \mathrm{Ab} \text { region }\end{array}$ & No & $\mathrm{C}>\mathrm{T}$ chr21:27394285 & 45.8 & NA & Possibly damaging & \\
\hline C06-25448 & $\mathrm{AD}$ & PSEN2 & S130L & rs63750197 & Known & No & $\mathrm{C}>\mathrm{T}$ chr1:227073271 & 46.7 & $>0.1$ & Possibly damaging & hippo \\
\hline C08-19292 & $\mathrm{CT}$ & MAPT & $\mathrm{P} 4 \mathrm{~T}$ & & NOVEL & No & C $>$ A chr17:44039713 & 39.8 & n.a. & Probably damaging & cer \\
\hline BBN_16281 & $\mathrm{CT}$ & APP & A479S & rs143794560 & $\begin{array}{l}\text { Outside of } \mathrm{Ab} \text { region } \\
\text { outside of } \mathrm{Ab} \text { region }\end{array}$ & No & C > A chr21:27347406 & 44.4 & NA & Benign & cer \\
\hline BBN_16213 & $\mathrm{CT}$ & PSEN2 & D439A & rs63750110 & Known & No & A $>$ C chr1:227083249 & 43.0 & NA & Probably damaging & cer \\
\hline UK82 & $\mathrm{CT}$ & PSEN2 & $\mathrm{A} 258 \mathrm{~T}$ & rs148238688 & & No & $\mathrm{G}>\mathrm{A} \operatorname{chr} 1: 227076735$ & 52.2 & NA & Probably damaging & blood \\
\hline BBN_10197 & $\mathrm{AD}$ & MAPT & IVS10 + 29 & rs63751443 & Not pathogenic & None recorded & $\mathrm{G}>\mathrm{A} \operatorname{chr} 17: 44087797$ & 48.8 & NA & NA & \\
\hline C08-31992 & $\mathrm{AD}$ & MAPT & IVS10 + 29 & rs63751443 & Not pathogenic & None recorded & $\mathrm{G}>\mathrm{A} \operatorname{chr} 17: 44087797$ & 46.3 & NA & NA & \\
\hline BBN_9967 & $\mathrm{AD}$ & PSEN1 & E318G & rs 362373 & Not pathogenic & No & $A>G$ chr14:73673178 & 53.7 & 0.9 & Benign & \\
\hline BBN_18399 & $\mathrm{CT}$ & PSEN1 & E318G & rs362373 & Not pathogenic & None recorded & $A>G$ chr14:73673178 & 49.1 & 0.9 & Benign & \\
\hline BBN_9952 & $\mathrm{AD}$ & MAPT & V224G & rs141120474 & Possible risk factor & None recorded & $\mathrm{T}>\mathrm{G} \operatorname{chr} 17: 44060841$ & 41.0 & $>0.1$ & Possibly damaging & \\
\hline C09-20305 & $\mathrm{AD}$ & MAPT & $\mathrm{A} 469 \mathrm{~T}$ & rs143624519 & Possible risk factor & No & $\mathrm{G}>\mathrm{A} \operatorname{chr} 17: 44068850$ & 46.7 & 0.2 & Benign & \\
\hline BBN_9959 & $\mathrm{AD}$ & MAPT & $\mathrm{S} 427 \mathrm{~F}$ & rs143956882 & Possible risk factor & None recorded & $\mathrm{C}>\mathrm{T} \operatorname{chr} 17: 44067341$ & 44.3 & $>0.1$ & Probably damaging & \\
\hline C08-07965 & $\mathrm{CT}$ & MAPT & V224G & rs141120474 & Possible risk factor & None recorded & $\mathrm{T}>\mathrm{G} \operatorname{chr} 17: 44060841$ & 42.4 & $>0.1$ & Possibly damaging & \\
\hline C06-29159 & $\mathrm{CT}$ & MAPT & S427F & rs143956882 & Possible risk factor & None recorded & $\mathrm{C}>\mathrm{T} \operatorname{chr} 17: 44067341$ & 52.5 & $>0.1$ & Probably damaging & \\
\hline
\end{tabular}

NOTE. For each of the 21 validated heterozygous variants (excluding known nonpathogenic MAPT polymorphisms), we report the information on the subject (diagnostic group, family history of AD) and the

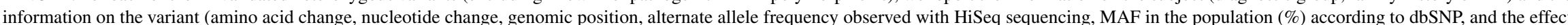

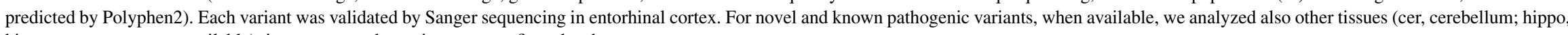
hippocampus; n.a., not available), in every case the variant was confirmed as heterozygous. 
964

965

966

967

968

969

970

971

972

973

974

975

976

977

978

979

980

981

982

983

984

985

986

987 눈

988 ن

989 응

990

991

992

993

994

995

996

997

998

999

1000

1001

1002

1003

1004

1005

1006

1007

1008

1009

1010

1011

1012

1013

1014

1015

1016

1017

1018

1019

1020

1021

1022

1023

1024

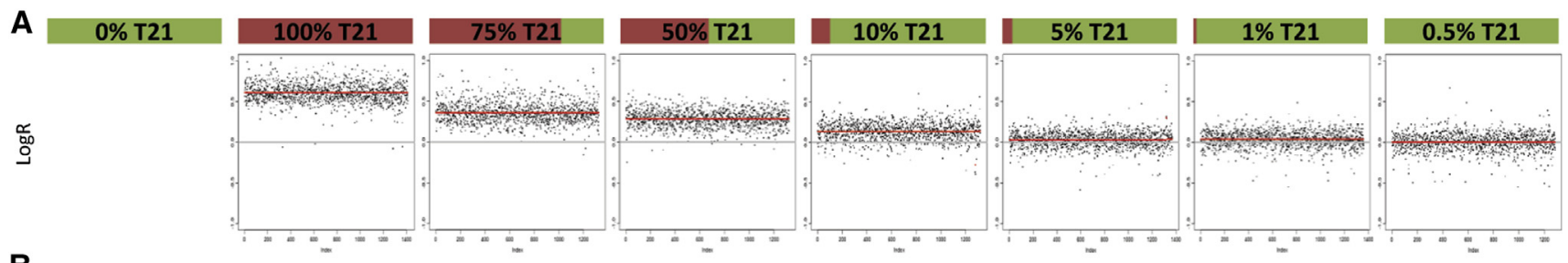

B
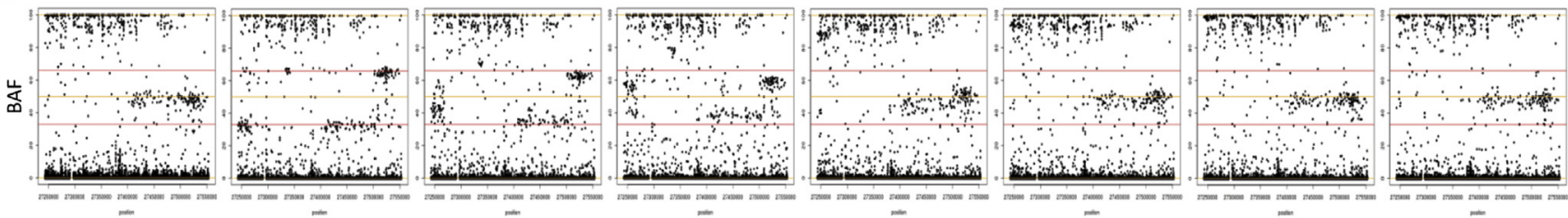

C

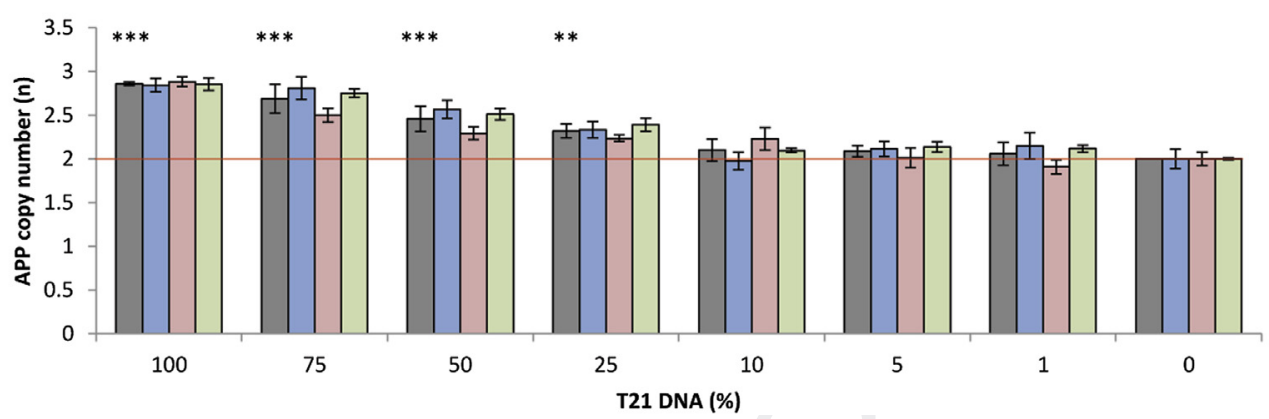

$\square 3$ assays average

口Hs01180853_cn

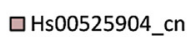

口Hs05547973_cn

Fig. 4. Deep sequencing of capture-enriched libraries allows the detection of $10 \%$ mosaic copy number variants (CNVs). A series of mixtures of DNA from a trisomy 21 (T21) subject and from an euploid subject was analyzed to assess the lower limit of mosaic CNV detection. For each sample in the series (indicated with colored bars), we report the normalized sequencing depth compared with the diploid control (LogR, A) and the B-allele frequency (BAF, B), calculated for all positions of the captured region. The region presented on the $\mathrm{x}$ axis in both (A) and (B) corresponds to the captured APP locus (chr21:27242859-27553138). Detection of mosaic CNVs was also performed by qPCR using three different probe sets (C). The calculated copy number of the $A P P$ gene for each sample is reported for each probe set used and as an average of the signal obtained from the three probes for each sample. Asterisks denote statistical significant differences versus the euploid sample $(* * * P<.0001, * * P<.001)$.

analysis with the $\log \mathrm{R}$ method is the most sensitive method to detect mosaic CNVs.

We analyzed accordingly our sequencing data, but no CNVs of APP, PSEN1, PSEN2, or MAPT were found. These results indicate that the brain samples analyzed had more than $90 \%$ of cells with the correct number of copies of these genes.

\section{Discussion}

The potential contribution of somatic mutagenesis to neurodegenerative disorders is increasingly recognized, but little systematic study of this problem is available. Here, we developed a methodology to analyze the presence of somatic mutations in known FAD genes in the brain. Using a library enrichment-deep sequencing method we were able to simultaneously interrogate the presence of mosaic SNVs having a mutant allele frequency of $1 \%$ or more, and mosaic CNVs present in as few as $10 \%$ of cells.

Although our method of library enrichment for a $\sim 600$ $\mathrm{kb}$ ROI allows the simultaneous query of mosaic SNVs and CNVs, based on the analysis of "synthetic mosaic" samples, it is clear that an allele frequency of $1 \%$ is an absolute limit for mosaic SNVs detection at high sensitivity and workable specificity, due to increasingly FP calls. Amplicon-based ultradeep resequencing with MiSeq was sensitive enough to detect "synthetic mosaic" variants having $0.5 \%$ mutant allele frequency and enabled the validation of the putative variants. Further studies aimed at the detection of mosaic SNVs from a selected number of genes or exons could thus be efficiently and cost-effectively performed by deep-sequencing of PCR amplicons.

In this pilot study we identified three subjects, two confirmed SAD and one Braak II "control", with MiSeqvalidated mosaic variants. Two of these mosaic variants were novel mutations of MAPT of unknown pathogenicity. Bioinformatic prediction by Polyphen2 [39] indicates that both MAPT Q124K and MAPT S400A (Tau-F numbering) are "probably damaging". MAPT Q124K is located in the $\mathrm{N}$-terminus of tau, whereas all known pathogenic tau mutations concentrate in the microtubule-binding domains of the C-terminus. In contrast, MAPT S400A is located in the Cterminus close to a known pathogenic mutation (R406W). Although MAPT mutations do not cause familial AD, based on the "double hit" cascade proposed for late onset AD [40] we suggest the possibility that mosaic MAPT mutation may co-operate with imbalances in $A \beta$ metabolism (for instance, age-associated clearance problems with $A \beta$ ). Given the 
limited number of positive data, we cannot at the moment make any statistical valid prediction about the possible association of such mutations with sporadic AD. The third mosaic mutation, PSEN2 S130L, has been found before in an AD family [41] and was also found in an SAD case [42]. The pathogenicity of the PSEN2 S130L is disputed, as it has been also found in two healthy individuals, however, we note that in one instance the healthy subject was younger than the age of onset for this particular mutation [41], and in the second instance age was not disclosed [42]. In our cohort, we found the PSEN2 S130L variant as a nonmosaic heterozygous mutation also in a SAD patient, who displayed an age of onset at 66 years and died at 88 years of age. Finally, we note that this mutation is predicted "possibly damaging" by Polyphen2 and that it is located in the first loop of PSEN2, next to three other pathogenic mutations (T122P, T122R, E126K), however, pathogenicity of this mutation is clearly not established $[42,43]$.

Recent studies have highlighted that pathogenic variants in AD-related genes can indeed be found in apparently sporadic $\mathrm{AD}$ cases, both early [44,45] and late onset [43]. Accordingly, in our study we found a relatively high number of heterozygous mutations in brains from SAD patients. Among these, six variants deserve some further discussion: one confirmed pathogenic mutation (PSEN1 H163R [46]), two variants of uncertain pathogenicity (PSEN2 R62C and PSEN2 S130L), and three novel variants of unknown pathogenicity (PSEN2 V101M, PSEN2 L238F, and MAPT A90V). Both Polyphen2 and SIFT [47] predict that PSEN2 V101M and PSEN2 L238F are "probably damaging" and "damaging" (SIFT scores 0 and 0.05 , respectively), whereas MAPT A90V is unlikely pathogenic (predicted "benign" by Polyphen2). These six mutations were heterozygous in both entorhinal cortex and cerebellum, indicating that they are most likely constitutive variants rather than mosaics. Lack of DNA samples from the parents prevented the investigation of whether the mutations occurred de novo in the germline, but clinically the six cases were reported as apparent sporadic. The parents of the patient bearing the pathogenic PSEN1 H163R (rs63750590) [46,48] mutation died at 66 and 72 years, respectively, from heart problems and did not show any signs of cognitive decline, whereas the patient had an onset at 51 years and died at 56 years, hinting that this mutation may have appeared de novo. Further efficient study of mosaic pathogenic variants in neurodegenerative disease requires that brain banks also ascertain access to DNA from peripheral blood and, if possible, to DNA from the close relatives.

We profited from our data set to try to uncover possible mosaic APP duplications, as this is a known cause of FAD [49]. Although in this pilot experiment no duplication of the APP locus was found, we notice that our method allows detecting a $10 \%$ mosaic gain and is therefore at least twice as sensitive as quantitative PCR-based approaches.

Our study demonstrates that the analysis of brain samples (as opposed to blood samples) could provide unexpected new insights into the possibility that mosaics contribute to the risk of developing AD. Here, we chose to study entorhinal cortex, as this is the area where the first tau aggregates appear over the course of AD pathology [50]. Because mutant cells in this area may have been lost rather early in the disease process, follow-up studies should also sample other brain areas, to explore in a more systematic way the phenomenon of mosaicisms for these genes. Moreover, it will be important to study brain samples patients from whom blood and possibly gDNA from both parents are available.

In conclusion, we show that variants in AD-related genes with low allele frequencies can be detected in brain-derived DNA. Although our data cannot formally prove the pathogenicity of the mosaic variants identified, our work prompts for follow-up studies in larger cohorts and using multiple sampling of the same brain to understand whether mosaic mutations might be causally linked to the disease. In fact, and a priori, somatic genetic mosaicism may prove to have a larger effect on disease etiology than common susceptibility factors identified via genome-wide association studies. Our work shows the feasibility of a larger and systematic study to confirm or refute the hypothesis of mosaic mutations as a cause of sporadic AD.

\section{Acknowledgments}

The authors thank Dr. Jeroen Van Houdt, the staff of the UZ Genomic Core and Dr. Moritz Gerstung for useful discussions. The authors also thank Dr. Peter Verhasselt of VIB Nucleomics Core for help with design of the indexed primers for MiSeq sequencing. We thank Prof. Hilde van Esch for providing trisomy 21 amniocytes and Prof. Rik Van den Berghe for the fibroblasts of patient bearing the APP E682K mutation (APP mutant). The authors thank the many anonymous patients who donated their brain to the different brain banks that provided material for this study (Lille NeuroBank and from the London Neurodegenerative Diseases Brain Bank).

Funding: The research was supported by a grant from the Fonds voor wetenschappelijk onderzoek (FWO) to BDS, TV, and PVL, and a Methusalem grant of the Flemish Government and an Advanced ERC grant, both to BDS. BDS is Arthur Bax-Anna Van Luffelen Professor for Alzheimer's Disease. CSF was supported by a Marie Curie IntraEuropean Fellowship. PL was supported by the COEN Pathfinder initiative (NEURO-MIR). London Neurodegenerative Diseases Brain Bank received funding from the MRC (Medical Research Council) and from the Brains for Dementia Research project (funded by Alzheimer's Society and Alzheimer's Research UK).

\section{Supplementary data}

Supplementary data related to this article can be found at http://dx.doi.org/10.1016/j.jalz.2015.02.007. 


\section{RESEARCH IN CONTEXT}

1. Systematic review: Postzygotic acquired (somatic) mutations in Alzheimer's disease (AD)-relevant genes could originate small group of brain cells producing pathogenic amyloid beta $(\mathrm{A} \beta)$ and Tau aggregates which could spread over the brain, thus causing sporadic AD. "Prion-like" spreading and seeding of both $A \beta$ and Tau aggregates has been documented in vitro and in vivo.

2. Interpretation: We describe a sensitive method to detect and validate low-allele frequency mosaic mutations. Moreover we report the discovery of putative mosaic mutations in brain-derived DNA of AD patients and controls.

3. Future directions: The full extent of pathogenic mosaic mutations in brain will be clear on the examination of multiple brain areas in wider cohorts of subjects. Importantly, the concept of pathogenic mosaic mutations can be explored also in other neurodegenerative diseases, such as Parkinson's disease.

\section{References}

[1] Watson IR, Takahashi K, Futreal PA, Chin L. Emerging patterns of somatic mutations in cancer. Nat Rev Genet 2013;14:703-18.

[2] Stratton MR, Campbell PJ, Futreal PA. The cancer genome. Nature 2009;458:719-24.

[3] Yates LR, Campbell PJ. Evolution of the cancer genome. Nat Rev Genet 2014;13:795-806.

[4] Lee JH, Huynh M, Silhavy JL, Kim S, Dixon-Salazar T, Heiberg A, et al. De novo somatic mutations in components of the PI3K-AKT3mTOR pathway cause hemimegalencephaly. Nat Genet 2012; 44:941-5.

[5] Poduri A, Evrony GD, Cai X, Elhosary PC, Beroukhim R, Lehtinen MK, et al. Somatic activation of AKT3 causes hemispheric developmental brain malformations. Neuron 2012;74:41-8.

[6] Jamuar SS, Lam AT, Kircher M, D’Gama AM, Wang J, Barry BJ, et al. Somatic mutations in cerebral cortical malformations. N Engl J Med 2014;371:733-43.

[7] Hu WF, Chahrour MH, Walsh CA. The diverse genetic landscape of neurodevelopmental disorders. Annu Rev Genomics Hum Genet 2014;15:195-213.

[8] Frank SA. Evolution in health and medicine Sackler colloquium: somatic evolutionary genomics: mutations during development cause highly variable genetic mosaicism with risk of cancer and neurodegeneration. Proc Natl Acad Sci U S A 2010;107(Suppl 1):1725-30.

[9] Pamphlett R. Somatic mutation: a cause of sporadic neurodegenerative diseases? Med Hypotheses 2004;62:679-82.

[10] Poduri A, Evrony GD, Cai X, Walsh CA. Somatic mutation, genomic variation, and neurological disease. Science 2013;341:1237758.

[11] Proukakis C, Houlden H, Schapira AH. Somatic alpha-synuclein mutations in Parkinson's disease: hypothesis and preliminary data. Mov Disord 2013;28:705-12.
[12] Kingsbury MA, Friedman B, McConnell MJ, Rehen SK, Yang AH, Kaushal D, et al. Aneuploid neurons are functionally active and integrated into brain circuitry. Proc Natl Acad Sci U S A 2005; 102:6143-7.

[13] Muotri AR, Gage FH. Generation of neuronal variability and complexity. Nature 2006;441:1087-93.

[14] Cai X, Evrony GD, Lehmann HS, Elhosary PC, Mehta BK, Poduri A, et al. Single-cell, genome-wide sequencing identifies clonal somatic copy-number variation in the human brain. Cell Rep 2014;8:1280-9.

[15] Nussbaum R, McInnes R, Willard H. Thompson \& Thompson genetics in medicine. 7th Edition. Elsevier; 2007.

[16] Behjati S, Huch M, van Boxtel R, Karthaus W, Wedge DC, Tamuri AU, et al. Genome sequencing of normal cells reveals developmental lineages and mutational processes. Nature 2014;513:422-5.

[17] Azevedo FA, Carvalho LR, Grinberg LT, Farfel JM, Ferretti RE, Leite RE, et al. Equal numbers of neuronal and nonneuronal cells make the human brain an isometrically scaled-up primate brain. $\mathbf{J}$ Comp Neurol 2009;513:532-41.

[18] Beck JA, Poulter M, Campbell TA, Uphill JB, Adamson G, Geddes JF, et al. Somatic and germline mosaicism in sporadic early-onset Alzheimer's disease. Hum Mol Genet 2004;13:1219-24.

[19] Campion D, Dumanchin C, Hannequin D, Dubois B, Belliard S, Puel M, et al. Early-onset autosomal dominant Alzheimer disease: prevalence, genetic heterogeneity, and mutation spectrum. Am J Hum Genet 1999;65:664-70.

[20] Bertram L, Tanzi RE. The genetics of Alzheimer's disease. Prog Mol Biol Transl Sci 2012;107:79-100.

[21] Aguzzi A, Rajendran L. The transcellular spread of cytosolic amyloids, prions, and prionoids. Neuron 2009;64:783-90.

[22] Kane MD, Lipinski WJ, Callahan MJ, Bian F, Durham RA, Schwarz RD, et al. Evidence for seeding of beta-amyloid by intracerebral infusion of Alzheimer brain extracts in beta-amyloid precursor protein-transgenic mice. J Neurosci 2000;20:3606-11.

[23] Eisele YS, Obermuller U, Heilbronner G, Baumann F, Kaeser SA, Wolburg $\mathrm{H}$, et al. Peripherally applied Abeta-containing inoculates induce cerebral beta-amyloidosis. Science 2010;330:980-2.

[24] Clavaguera F, Bolmont T, Crowther RA, Abramowski D, Frank S, Probst A, et al. Transmission and spreading of tauopathy in transgenic mouse brain. Nat Cell Biol 2009;11:909-13.

[25] de Calignon A, Polydoro M, Suarez-Calvet M, William C, Adamowicz DH, Kopeikina KJ, et al. Propagation of tau pathology in a model of early Alzheimer's disease. Neuron 2012;73:685-97.

[26] Tsiatis AC, Norris-Kirby A, Rich RG, Hafez MJ, Gocke CD, Eshleman JR, et al. Comparison of Sanger sequencing, pyrosequencing, and melting curve analysis for the detection of KRAS mutations: diagnostic and clinical implications. J Mol Diagn 2010;12:425-32.

[27] Li H, Durbin R. Fast and accurate short read alignment with BurrowsWheeler transform. Bioinformatics 2009;25:1754-60.

[28] Li H, Handsaker B, Wysoker A, Fennell T, Ruan J, Homer N, et al. The sequence alignment/map format and SAMtools. Bioinformatics 2009; 25:2078-9.

[29] McKenna A, Hanna M, Banks E, Sivachenko A, Cibulskis K, Kernytsky A, et al. The Genome Analysis Toolkit: a MapReduce framework for analyzing next-generation DNA sequencing data. Genome Res 2010;20:1297-303.

[30] Koboldt DC, Zhang Q, Larson DE, Shen D, McLellan MD, Lin L, et al. VarScan 2: somatic mutation and copy number alteration discovery in cancer by exome sequencing. Genome Res 2012;22:568-76.

[31] Wang K, Li M, Hakonarson H. ANNOVAR: functional annotation of genetic variants from high-throughput sequencing data. Nucleic Acids Res 2010;38:e164.

[32] Stead LF, Sutton KM, Taylor GR, Quirke P, Rabbitts P. Accurately identifying low-allelic fraction variants in single samples with nextgeneration sequencing: applications in tumor subclone resolution. Hum Mutat 2013;34:1432-8.

[33] Li H. Improving SNP discovery by base alignment quality. Bioinformatics 2011;27:1157-8. 
[34] Benitez BA, Karch CM, Cai Y, Jin SC, Cooper B, Carrell D, et al. The PSEN1, p.E318G variant increases the risk of Alzheimer's disease in APOE-epsilon4 carriers. PLoS Genet 2013;9:e1003685.

[35] Cruchaga C, Haller G, Chakraverty S, Mayo K, Vallania FL, Mitra RD, et al. Rare variants in APP, PSEN1 and PSEN2 increase risk for AD in late-onset Alzheimer's disease families. PLoS One 2012;7:e31039.

[36] D'Souza I, Poorkaj P, Hong M, Nochlin D, Lee VM, Bird TD, et al. Missense and silent tau gene mutations cause frontotemporal dementia with parkinsonism-chromosome 17 type, by affecting multiple alternative RNA splicing regulatory elements. Proc Natl Acad Sci U S A 1999;96:5598-603.

[37] Biesecker LG, Spinner NB. A genomic view of mosaicism and human disease. Nat Rev Genet 2013;14:307-20.

[38] Conlin LK, Thiel BD, Bonnemann CG, Medne L, Ernst LM, Zackai EH, et al. Mechanisms of mosaicism, chimerism and uniparental disomy identified by single nucleotide polymorphism array analysis. Hum Mol Genet 2010;19:1263-75.

[39] Adzhubei IA, Schmidt S, Peshkin L, Ramensky VE, Gerasimova A, Bork $\mathrm{P}$, et al. A method and server for predicting damaging missense mutations. Nat Methods 2010;7:248-9.

[40] Small SA, Duff K. Linking Abeta and tau in late-onset Alzheimer's disease: a dual pathway hypothesis. Neuron 2008;60:534-42.

[41] Tedde A, Nacmias B, Ciantelli M, Forleo P, Cellini E, Bagnoli S, et al. Identification of new presenilin gene mutations in early-onset familial Alzheimer disease. Arch Neurol 2003;60:1541-4.

[42] Tomaino C, Bernardi L, Anfossi M, Costanzo A, Ferrise F, Gallo M, et al. Presenilin 2 Ser130Leu mutation in a case of late-onset "sporadic" Alzheimer's disease. J Neurol 2007;254:391-3. et al. Investigating the role of rare coding variability in Mendelian dementia genes (APP, PSEN1, PSEN2, GRN, MAPT, and PRNP) in late-onset Alzheimer's disease. Neurobiol Aging 2014;35: 2881.e1-6.

[44] Jin SC, Pastor P, Cooper B, Cervantes S, Benitez BA, Razquin C, et al. Pooled-DNA sequencing identifies novel causative variants in PSEN1, GRN and MAPT in a clinical early-onset and familial Alzheimer's disease Ibero-American cohort. Alzheimers Res Ther 2012;4:34.

[45] Sassi C, Guerreiro R, Gibbs R, Ding J, Lupton MK, Troakes C, et al. Exome sequencing identifies 2 novel presenilin 1 mutations (p.L166V and p.S230R) in British early-onset Alzheimer's disease. Neurobiol Aging 2014;35:2422.e13-6.

[46] Sherrington R, Rogaev EI, Liang Y, Rogaeva EA, Levesque G, Ikeda $\mathrm{M}$, et al. Cloning of a gene bearing missense mutations in early-onset familial Alzheimer's disease. Nature 1995;375:754-60.

[47] $\mathrm{Ng}$ PC, Henikoff S. Predicting deleterious amino acid substitutions. Genome Res 2001;11:863-74.

[48] Campion D, Flaman JM, Brice A, Hannequin D, Dubois B, Martin C, et al. Mutations of the presenilin I gene in families with early-onset Alzheimer's disease. Hum Mol Genet 1995;4:2373-7.

[49] Rovelet-Lecrux A, Frebourg T, Tuominen H, Majamaa K, Campion D, Remes AM. APP locus duplication in a Finnish family with dementia and intracerebral haemorrhage. J Neurol Neurosurg Psychiatry 2007; 78:1158-9.

[50] Braak H, Braak E. Neuropathological stageing of Alzheimer-related changes. Acta Neuropathol 1991;82:239-59.
[43] Sassi C, Guerreiro R, Gibbs R, Ding J, Lupton MK, Troakes C, 